UMN-TH-1415/95

astro-ph/9512034

submitted to The Astrophysical Journal

\title{
LiBeB PRODUCTION BY NUCLEI AND NEUTRINOS
}

\author{
Elisabeth Vangioni-Flam ${ }^{1}$, Michel Cassé ${ }^{2}$, Brian D. Fields ${ }^{1,4}$ and Keith A. Olive ${ }^{3}$ \\ ${ }^{1}$ Institut d'Astrophysique de Paris, CNRS \\ 98 bis bd Arago 75014 Paris, France \\ ${ }^{2}$ Service d'Astrophysique/DSM/DAPNIA/CEA \\ Saclay, France \\ ${ }^{3}$ School of Physics and Astronomy, University of Minnesota \\ Minneapolis, MN 55455, USA \\ ${ }^{4}$ Department of Physics, University of Notre Dame \\ Notre Dame, IN 46556
}

\begin{abstract}
The production of $\mathrm{LiBeB}$ isotopes by nuclear and neutrino spallation are compared in the framework of galactic evolutionary models. As motivated by $\gamma$-ray observations of Orion, different possible sources of low-energy $\mathrm{C}$ and $\mathrm{O}$ nuclei are considered, such as supernovae of various masses and WC stars. We confirm that the low energy nuclei (LEN), injected in molecular clouds by stellar winds and type II supernovae originating from the most massive progenitors, can very naturally reproduce the observed Be and B evolution in the early galaxy (halo phase). Assuming the global importance of the LEN component, we compute upper and lower bounds to the neutrino process contribution corresponding to limiting cases of LEN particle spectra. A consistent solution is found with a spectrum of the kind proposed by Ramaty et al. (1995a,b), e.g. flat up to $E_{c}=30 \mathrm{MeV} / \mathrm{n}$ and decreasing abruptly above. This
\end{abstract}


solution fulfills the challenge of explaining at the same time the general Be and B evolution, and their solar system abundances without overproducing ${ }^{7} \mathrm{Li}$ at very low metallicities, and the meteoritic ${ }^{11} \mathrm{~B} /{ }^{10} \mathrm{~B}$ ratio. In this case, neutrino spallation is constrained to play a limited role in the genesis of the solar system ${ }^{11} \mathrm{~B}$. Galactic cosmic rays (GCR) become operative late in the evolution of the disk $([\mathrm{Fe} / \mathrm{H}]>-1)$, but their contribution to the solar abundances of ${ }^{9} \mathrm{Be},{ }^{10} \mathrm{~B}$ and ${ }^{11} \mathrm{~B}$ is not dominant $(35 \%, 30 \%$ and $20 \%$ respectively). Thus, with this LEN spectrum, GCR are not the main source of ${ }^{9} \mathrm{Be}$ and $\mathrm{B}$ in the Galaxy. The most favorable case for neutrinos, (adopting the same kind of spectrum) has $E_{c}=20 \mathrm{MeV} / \mathrm{n}$. Even in this case, the neutrino yields of Woosley and Weaver (1995) must to be reduced by a factor of 5 to avoid ${ }^{11} \mathrm{~B}$ overproduction. Furthermore, this solution leads to a high $\mathrm{B} / \mathrm{Be}$ ratio at $[\mathrm{Fe} / \mathrm{H}]=-$ 2 , difficult to reconcile with the observations, unless specifically non-local thermodynamic equilibrium corrections to the boron abundance are large. On the other hand, if neutrino spallation does play an important role in the production of galactic ${ }^{11} \mathrm{~B}$, then LEN processes are relegated to a more local phenomenon. However, in this case, unless neutrino spallation can also produce ${ }^{9} \mathrm{Be}$ (and to some extent ${ }^{10} \mathrm{~B}$ and ${ }^{6} \mathrm{Li}$ ), a new source of primary ${ }^{9} \mathrm{Be}$ must be found. 


\section{Introduction}

Since the 1970's it has been known that spallation reactions between high-energy nuclei in the galactic cosmic radiation (GCR) and the interstellar medium are an important source of $\mathrm{Li}, \mathrm{Be}$, and $\mathrm{B}(\mathrm{LiBeB})$ isotopes. However, this mechanism is known to be incomplete, as indicated by the well-established failure of GCR production to reproduce the ${ }^{7} \mathrm{Li}$ abundance in Population I stars, the meteoritic ${ }^{11} \mathrm{~B} /{ }^{10} \mathrm{~B}$ isotopic ratio, and as shown more recently by the linear (rather than quadratic) proportionality between Be (and B) and Fe in the early Galaxy (Population II). Thus there is a need for an additional source of LiBeB. Partly in response to this need, two new sources of $\mathrm{LiBeB}$, and particularly ${ }^{11} \mathrm{~B}$ have been recently proposed:

1. Synthesis of ${ }^{7} \mathrm{Li}$ and ${ }^{11} \mathrm{~B}$ by neutrino spallation in the helium and carbon shells of supernovae (Woosley et al. 1990, Olive et al. 1994, Timmes et al. 1995)-the so-called " $\nu$-process."

2. Breakup of low energy nuclei (LEN) injected in molecular clouds of the kind detected in Orion through their gamma ray line emission (Bloemen et al. 1994, Cassé et al. 1995, Ramaty et al. 1995a,b,1996, Fields et al. 1995a).

With the addition of these processes, spallation now depends on three sources related to high energy $(>500 \mathrm{MeV} / \mathrm{n})$ and low energy $(<30 \mathrm{MeV} / \mathrm{n})$ nuclei, and to neutrinos $(<8 \mathrm{MeV})$. The effect of the $\nu$-process on LiBeB evolution has been considered by Olive et al. (1994). These authors followed Prantzos et al. (1993) in assuming that GCR were more efficiently confined in the early galaxy. By itself, such an "overconfinement" model reproduced crudely the Be and B evolutions (but not perfectly, see Tayler 1995). The most recent data confirm the linearity of both the B and ${ }^{9} \mathrm{Be} v s$. Fe correlations (Duncan et al. 1995; Rebull et al. 1995). As such they are indeed difficult to reconcile with the overconfinement model, especially at very low metallicity where the calculated slope is 2. Moreover, the ${ }^{11} \mathrm{~B} /{ }^{10} \mathrm{~B}$ ratio always remains unavoidably close to 2.5 , the usual GCR value. To obtain a solar boron isotopic ratio of ${ }^{11} \mathrm{~B} /{ }^{10} \mathrm{~B}=4.05 \pm 0.05$ (Shima and Honda 1962, Chaussidon \& 
Robert 1995), Olive et al. (1994) introduced neutrino spallation, which enhances ${ }^{11} \mathrm{~B}$ (and not ${ }^{10} \mathrm{~B}$ ) and so can give the right boron isotopic ratio in the solar system. Since this process produces negligible amounts of ${ }^{9} \mathrm{Be}$, one has to invoke a separate production of this isotope by nuclear spallation.

Recently, the $\nu$-process yields have been updated (Woosley \& Weaver 1995), and more importantly, the Bloemen et al. (1994) discovery of unexpected $\gamma$-ray emission from Orion has provided strong evidence for a LEN component. The Orion $\gamma$-radiation is consistent with line emission from ${ }^{12} \mathrm{C}^{*}$ and ${ }^{16} \mathrm{O}^{*}$ and as such can only be plausibly explained by a large flux of low-energy nuclei enriched in $\mathrm{C}$ and $\mathrm{O}$. Given this observational evidence for LEN, and its ability to copiously produce $\mathrm{LiBeB}$, the combined effect with neutrino spallation should be reconsidered. In this paper we will explore constraints on the relative contribution of all three galactic LiBeB sources: GCR, LEN, and the $\nu$ - process. We will show that one can significantly constrain the relative contribution of the different sources by exploiting the particularities of each process and the interplay of these components in LiBeB evolution.

The paper is organized logically as follows: we first introduce the three components of LiBeB production and discuss the available free parameters for each. In particular, since the origin of the LEN is still debated, as are the corresponding LEN source abundances, we introduce various $\mathrm{C}$ and $\mathrm{O}$ enriched compositions related to stellar winds and supernovae of different masses. Next, the parameters for the different production mechanisms are constrained by comparing to the available observational and solar system data on LiBeB evolution. These data consist of the history of the elemental abundances (observed as a function of metallicity $[\mathrm{Fe} / \mathrm{H}])$ and of the solar system isotopic abundances and their ratios. Specifically, the adopted solutions are bound by observational requirements: 1) to avoid Li overproduction at low metallicity (i.e. preserve the Spite plateau, e.g. Spite and Spite 1993); 2) to reproduce the quasi linear relationship between $\mathrm{Be} / \mathrm{H}$ and $\mathrm{B} / \mathrm{H}$ vs. $[\mathrm{Fe} / \mathrm{H}]$, at least up to $[\mathrm{Fe} / \mathrm{H}]<-1 ; 3)$ to reproduce the solar $\mathrm{Be} / \mathrm{H}$ abundance; 4) to generate a ${ }^{11} \mathrm{~B} /{ }^{10} \mathrm{~B}$ ratio such that, after mixing with the GCR products $\left({ }^{11} \mathrm{~B} /{ }^{10} \mathrm{~B}=2.5\right)$ and the neutrino induced ${ }^{11} \mathrm{~B}$ we get the observed meteoritic value of ${ }^{11} \mathrm{~B} /{ }^{10} \mathrm{~B}=4.05 \pm 0.05$ (Chaussidon and Robert 1995). 
We evaluate the possible models, showing that each process contributes in a unique way to LiBeB evolution, and that the different behaviors of the proposed mechanisms allow only a restricted set of possible combinations of them that still fit the data. In particular, we will show that if the LEN component produces Be and B in the early Galaxy as observed in Pop II stars, then this mechanism remains today the dominant source of LiBeB, contrary to what has been previously thought. Also, as a continuation of the work of Olive et al. (1994), we determine the maximum contribution of the neutrino process to the LiBeB production in the new context in which LEN are considered.

Two important assumptions should be made explicit here. (1) We take the LEN component, observed to exist in Orion, to be ubiquitous to all star-forming regions throughout the Galaxy's history. Also, (2) we determine the LEN irradiation time such that the particle bombardment in these regions gives the LiBeB abundances observed in extreme Pop II stars. As we will argue, the required exposure times ( $\lesssim 10^{5} \mathrm{yr}$ ) are reasonable.

A word about the chemical evolution models is in order. We adopt the same standard galactic evolutionary model as in Olive et al. (1994) and Vangioni-Flam et al. (1995). Our analysis is restricted to a simple closed box model including production of light elements by GCR, LEN and neutrinos, and their destruction in stars, as in Olive et al. (1994). We take into account the new neutrino yields released by Woosley and Weaver (1995) and recent work on the low energy nuclei injected in active star forming regions by Cassé et al. (1995), Vangioni-Flam et al. (1995) and Ramaty et al. (1995a,b, 1996), analyzing in finer details SN of different masses.

\section{The role of the different components}

\subsection{LEN}

The nuclear lines observed in Orion (Bloemen et al. 1994) must be excited via low-energy nuclear interactions; the $\gamma$-ray emission thus provides the first direct evidence of a LEN component. As the astrophysical source of LEN is uncertain, we will try three different models ( $\S 3.1-3.3)$. The first of these models to have been considered is the one most studied 
in the literature, and the one that does the best job of giving LiBeB evolution; we will thus focus on this model for the moment. In this scenario, the production rates of the LiBeB isotopes have been determined adopting a simple source spectrum of the kind $N(E)=$ const up to $E=E_{c}$, and $N(E) \propto E^{-n}$ above $E_{c}$. We follow Cassé et al. (1995), and Ramaty et al. (1995a,b) in assuming all nuclear species to have the same source spectrum, which we propagate down to thermalization in the cloud. We assume that Orion is representative of all the active star forming regions in the galaxy at all times; consequently the production rates need to be multiplied by the irradiation or exposure time $\tau$ to get the corresponding yields. The LEN mechanism is thus determined by a source composition (or - if one assumes the sources to come from a variety of supernovae- the lower limit $m_{\text {inf }}$ of this spectrum), the exposure time $\tau$, and the spectral parameters $n$ and $E_{c}$. (A summary of the production mechanisms, the free parameters in each, and how these are fit is found in Tables 1 and 2.)

The LEN component is uniquely capable of naturally producing significant Be in the early Galaxy, and moreover it naturally gives the observed approximately linear relation between Be (as well as B) and $\mathrm{Fe} / \mathrm{H}$. We will thus require that LEN reproduces the early galactic Be; having done so, the yields of Li and B will depend on the particular LEN particle spectrum chosen. Thus the details of the LEN contribution (and thus of the GCR and $\nu$ process contributions) to LiBeB analysis depend upon the spectrum assumed, and so some investigation of spectral dependence is in order.

For the present work, however, rather than examining the full parameter space of possible LEN spectra, i.e., of $\left(n, E_{c}\right)$ pairs, we will instead focus on two interesting limiting cases. The first is the case emphasized in the literature so far, in which one searches for spectra which give LEN nucleosynthesis yields consistent with all the constraints discussed above. This will have the effect of minimizing the need for contributions from the other sources, in particular from the $\nu$-process. The LEN spectrum for this case was first sought by Cassé $e t$ al. (1995), who favored an $E_{c} \sim 10 \mathrm{MeV}$. Since then it has been pointed out (Ramaty et al. 1995a, b) that energetic considerations favor $E_{c} \sim 30 \mathrm{MeV}$. We will examine this more recent model, which has an index $n=9$.

Secondly, we will consider the case of the spectrum which has the lowest possible contribution of ${ }^{11} \mathrm{~B}$ given the $\mathrm{Be}$ production (i.e., the lowest ${ }^{11} \mathrm{~B} / \mathrm{Be}$ ratio) corresponding to 
$E_{c}=20 \mathrm{MeV} / \mathrm{n}$ (also with $n=9$ ). This gives the maximal room for a $\nu$-process contribution. Thus this will set an upper limit to the needed $\nu$-process yields for LEN-dominated LiBeB evolution.

For a given spectrum, the remaining parameters for the LEN component are the composition and the irradiation time $\tau$. We will try different compositions, both of particular kinds of supernovae (i.e., those with Wolf-Rayet progenitors), and of ensembles of supernovae of different masses. In our analysis we will adjust $\tau$ to fit the observed rise of $\mathrm{Be} / \mathrm{H}$ at low $[\mathrm{Fe} / \mathrm{H}]$. Note that $\mathrm{B} / \mathrm{H}$ in this metallicity regime is thus fixed, and becomes a prediction of the model. The contribution of LEN at higher metallicity is judged by comparison with the observed ratios.

\subsection{Galactic Cosmic Rays}

The GCR is treated classically (i.e., without the overconfinement used in Prantzos et al. 1993). The relative production of the different isotopes is taken from the rates given by Read \& Viola (1984); their intensity is taken proportional to the supernova rate and is taken as a free parameter. Since the $\nu$-process does not produce Be, for a given LEN production rate, the GCR component must add whatever extra Be is necessary to obtain the solar abundance. Thus the GCR intensity is fixed to obtain the right $\mathrm{Be} / \mathrm{H}$ at solar birth, adding their contribution to the LEN one. (Fitting the intensity is equivalent to determining the present effective cosmic ray flux $F$; the implications of this point are further discussed in Lemoine et al. 1995). Having fixed the GCR contribution to give the correct solar Be abundance, the abundances of ${ }^{10} \mathrm{~B}$ and ${ }^{11} \mathrm{~B}$ become predicitions of the model.

\section{$2.3 \quad$ Neutrinos}

Neutrino spallation is a source of ${ }^{7} \mathrm{Li}$ and ${ }^{11} \mathrm{~B}$ via the interactions of neutrinos (predominantly $\nu_{\mu}$ and $\nu_{\tau}$ ) on nuclei, and of particular importance here on ${ }^{4} \mathrm{He}$ and ${ }^{12} \mathrm{C}$ (Woosley et al. 1990, Woosley and Weaver 1995 - hereafter WW). The lithium and boron yields are quite sensitive to the temperature of the $\mu$ and $\tau$ neutrinos, in which there is a fair amount of uncertainty (e.g. Janka \& Muller 1995). As a result, the overall yields of ${ }^{7} \mathrm{Li}$ and ${ }^{11} \mathrm{~B}$ have considerable 
uncertainties and we will consider variations in the overall production level. In Olive et al. (1994), $\nu$-process nucleosynthesis was incorporated into a model of galactic chemical evolution with the primary purpose of augmenting the low value for ${ }^{11} \mathrm{~B} /{ }^{10} \mathrm{~B}$ produced by standard GCR nucleosynthesis. To correctly fit the observed ratio of 4, it was found that the yields of Woosley et al. (1990) had to be tuned down by a factor of about 2 to avoid the overproduction of ${ }^{11} \mathrm{~B}$. Tuning down the $\nu$-process yields ensured as well that the production of ${ }^{7} \mathrm{Li}$ was insignificant, and did not affect the Spite plateau. However, the resulting ratio of $\mathrm{B} / \mathrm{Be}$ was predicted not only to be high, but also to be metallicity dependent with $\mathrm{B} / \mathrm{Be}$ increasing at low metallicity. The recently non-LTE corrected (Kiselman 1994; Kiselman \& Carlsson 1994) B/Be ratio at low metallicity may lend support to the enhanced production of boron at early times (Fields, Olive \& Schramm, 1995b).

Recently, the $\nu$-process yields have been updated and now include a metallicity dependence (WW). We employ these new yields, but recognizing the uncertainties in the input physics (see 33.4 ) we allow the magnitude of the yields to be a relatively free parameter, scaling them by a factor $f$. Figures 1 and 2 show that the full yields $(f=1)$ lead to a marginal overproduction of ${ }^{7} \mathrm{Li}$ around $[\mathrm{Fe} / \mathrm{H}]=-1$; while ${ }^{11} \mathrm{~B}$ production by neutrinos is so efficient that no room is left for other mechanisms. This case is excluded since other processes are necessary to produce ${ }^{9} \mathrm{Be}$ which consequently introduce their own ${ }^{11} \mathrm{~B}$ contribution. If we reduce the yields by a factor of $2(f=0.5)$ (full lines in the figures), we can allow for other mechanisms contributing to the observed ${ }^{11} \mathrm{~B}$ abundance. Indeed, when the LEN processes are included to account for Population II ${ }^{9} \mathrm{Be}$, we will see that a further reduction in the $\nu$-process yields are necessary. Incidentally, note that at solar metallicity, the new yields are about twice the old ones. Olive et al. (1994) had divided the old yields by 2, which corresponds to a division by 4 of the new ones. In the discussion below, $f$ is adjusted to give the right ${ }^{11} \mathrm{~B} /{ }^{10} \mathrm{~B}$; once the $\mathrm{LEN}$ and $\mathrm{GCR}$ contributions have been fixed to fit the $\mathrm{Be}$ evolution, the $\mathrm{B}$ evolution in these models becomes a prediction. As we will see in the next section, when all the components are taken into account, the needed reduction in ${ }^{11} \mathrm{~B}$ yields will depend on the LEN spectrum adopted. 


\section{Evolution driven by typical LEN progenitors}

The calculation includes sequentially the three components: LEN to adjust the $\mathrm{Be} / \mathrm{H}$ evolution, GCR to fit the solar abundance of ${ }^{9} \mathrm{Be}$ and possibly neutrinos to explain the meteoritic ${ }^{11} \mathrm{~B} /{ }^{10} \mathrm{~B}$ ratio. This procedure will be applied in turn to the different LEN sources proposed: massive stars (typically $60 \mathrm{M}_{\odot}$ ) (Ramaty et al. 1995a,b, Vangioni-Flam et al. 1995); the external layers of type Ic supernovae (Fields et al. 1995); and ejecta of supernovae between 15 and $100 \mathrm{M}_{\odot}$, given high velocities in the explosion and then further reaccelerated in the local medium.

\subsection{The most massive stars}

The WC model adopted by Ramaty et al. (1995a,b, 1996) is one of the best LEN candidates. Moreover, including pre- and post-WC stages of evolution (O, Of, WN, and explosion) or considering the explosion of a massive star of $60 \mathrm{M}_{\odot}$, not affected by mass loss, as in the early Galaxy (Maeder 1992), we get results similar to the WC case (Vangioni-Flam et al. 1995). So the $\mathrm{WC}$ case will serve as a reference for all stars of $\sim 60 \mathrm{M}_{\odot}$ on the main sequence, independent of their metallicity and related mass loss.

As stated in $\$ 2.1$, we tried two limiting spectra. For the $E_{c}=30 \mathrm{MeV} / \mathrm{n}$ spectrum, the required irradiation time is $\tau=5 \times 10^{4}$ yr. A modest GCR contribution (35, 30 and $20 \%$ for ${ }^{9} \mathrm{Be},{ }^{10} \mathrm{~B}$ and ${ }^{11} \mathrm{~B}$ ), is sufficient to bring the calculated ${ }^{11} \mathrm{~B} /{ }^{10} \mathrm{~B}$ ratio at solar age to its observed value of $4.05 \pm 0.05$, leaving virtually no room for neutrino spallation. The ${ }^{11} \mathrm{~B} /{ }^{10} \mathrm{~B}$ ratio evolves from 4.8 (the LEN value) at the beginning of galactic evolution, to 4 at solar birth (fig. 3, full line). The derived B/Be ratio, evolving from 26 and 20, also agrees with observations at all metallicities (fig. 4, full line). Accordingly, in this case, the $\mathrm{WW}^{{ }^{11} \mathrm{~B}}$ neutrino yields are constrained to be reduced by a factor of at least $10(f=0.1)$ to avoid overproduction of this isotope.

The proposed solution, based on a WC composition and $E_{c}=30 \mathrm{MeV} / \mathrm{n}$ seems satisfactory since it explains at the same time the general ${ }^{9} \mathrm{Be}$ and $\mathrm{B}$ evolution without overproducing ${ }^{7} \mathrm{Li}$ in the early galaxy and the ${ }^{11} \mathrm{~B} /{ }^{10} \mathrm{~B}$ ratio in the solar system. However, a more involved solution, combining GCR, LEN and neutrinos cannot be a priori excluded. 
To explore the strength of the constraints on the $\nu$-process in our LEN-dominated scenario, we employed a LEN spectrum tuned to minimize ${ }^{11} \mathrm{~B}$ production, and thus leave maximal room for a $\nu$-process contribution, while still explaining the population II ${ }^{9}$ Be evolution. Indeed, the ${ }^{11} \mathrm{~B} /{ }^{10} \mathrm{~B}$ production ratio vs. $E_{c}$ for a WC composition (Ramaty et al. 1996) presents a minimum of about 3 at $20 \mathrm{MeV} / \mathrm{n}$ (compared to about 5 at $E_{c}=30 \mathrm{MeV} / \mathrm{n}$ at zero metallicity). Higher values of $E_{c}$ are excluded since they lead to line widths inconsistent with the COMPTEL data (Ramaty et al. 1995 a,b; Tatischeff et al. 1996). While this apparently excludes an $E_{c}$ as high as $100 \mathrm{MeV} / \mathrm{n}$ (for which the $\nu$-process contribution can be somewhat larger), it should be recalled that the line width data of Bloemen et al. (1993) are subject to some uncertainty.

Taking spectra with $E_{c}=20 \mathrm{MeV} / \mathrm{n}$, fitting the lower bound of the early Be evolution, and adjusting the cosmic ray flux to get the solar value of ${ }^{9} \mathrm{Be}$, we find an irradiation time of $5 \times 10^{4} \mathrm{yr}$ (this is equivalent to minimizing the the Orion contribution; see fig. 5.) In this case with $f=0.5$, we get a reasonable $\mathrm{Be}$ and $\mathrm{B}$ evolution, however we also get ${ }^{11} \mathrm{~B} /{ }^{10} \mathrm{~B}=7$ at the solar epoch which is far too large.

Therefore, we adopt $f=0.2$ which gives ${ }^{11} \mathrm{~B} /{ }^{10} \mathrm{~B}=4.5$, (fig. 3), which is close to the observed value. Any further reduction would lead us to the first case where neutrinos are negligible. For ${ }^{11} \mathrm{~B}$, the neutrino contribution in this case is $40 \%$, the GCR one is $25 \%$ and the Orion one is $35 \%$. Concerning ${ }^{9} \mathrm{Be}$, the respective contributions are $60 \%$ for GCR and $40 \%$ for Orion. Finally, for ${ }^{10} \mathrm{~B}$, we get $55 \%$ for GCR and $45 \%$ for Orion.

However, as shown in figure 4, the neutrino addition alters maximally the B/Be ratio at $[\mathrm{Fe} / \mathrm{H}]$ about -2 . Below this value, the Orion component dominates since it is produced by massive (fast evolving ) stars which eject their $\mathrm{C}$ and $\mathrm{O}$ very rapidly, whereas the neutrino component is delayed since it arises from all stars more massive than $11 \mathrm{M}_{\odot}$. Above $[\mathrm{Fe} / \mathrm{H}]$ $=-2$, the $\mathrm{B} / \mathrm{Be}$ ratio decreases due to the growing importance of GCR. This bump in the $\mathrm{B} / \mathrm{Be} v s .[\mathrm{Fe} / \mathrm{H}]$ curve provides a clear test to the operation of the neutrino mechanism in conjunction to the LEN process.

The recent data of Duncan et al. (1995), lead to a B/Be ratio of about 10, irrespective of $[\mathrm{Fe} / \mathrm{H}]$. Non-LTE corrections could enhance this ratio at low metallicity (Kiselman 1994; Kiselman \& Carlsson 1994; Thevenin et al. 1996). This correction, which is dependent on 
stellar parameters such as surface temperature and gravity, may be as large as a factor of 5 at $[\mathrm{Fe} / \mathrm{H}]=-2$. However, as shown in figure 4, there is a bump in the B/Be evolution and the NLTE correction decreases continuously with increasing metallicity. thus, the NLTE correction at $[\mathrm{Fe} / \mathrm{H}]=-2$ should not be greater than the correction at $[\mathrm{Fe} / \mathrm{H}]=-3$. This may be indicative of an intrinsic inconsistency between the neutrino and LEN processes. A measurement of ${ }^{11} \mathrm{~B} /{ }^{10} \mathrm{~B}$ in stars at this metallicity (which is not out of reach of the HST; Duncan 1995, private communication) would be of considerable interest since the predicted ratio is twice solar in this metallicity range, (fig.3).

The WC case posits that only very massive stars contribute to the LEN component, i.e., the lower limit $m_{\text {inf }}$ to the LEN sources is $m_{\mathrm{inf}}=60 \mathrm{M}_{\odot}$. Can other supernovae contribute to the LEN component? As the source of these particles remains unclear, this question remains an open one, but we can get an important hint by noting the Li/Be ratio produced by lower mass supernovae. For $m_{\text {inf }}<60 \mathrm{M}_{\odot}$, one has $\mathrm{Li} / \mathrm{Be} \gtrsim 100$; thus an attempt to fit the Be abundance at low metallicities will lead to a conflict with the Spite plateau. The high $\mathrm{Li} /$ Be production ratio can be understood physically as being due to the composition of the ejecta of lower-mass supernovae. Yields from these stars have large amounts of $\mathrm{H}$ and more importantly He, which is very effective, via the $\alpha+\alpha$ reaction, in producing Li. Thus it is clear that to have an acceptable Li production, such stars must be avoided and so $m_{\text {inf }}$ must be fairly large; in fact we find the lowest acceptable level is $m_{\text {inf }} \geq 35 \mathrm{M}_{\odot}$. This conclusion holds for both of the proposed spectra.

\subsection{The external layers of a SNIc}

If a supernova progenitor has been subject to heavy mass loss, then its outermost ejecta are propelled to high velocity corresponding to several MeV/n (Nomoto et al. 1995, Woosley, Langer and Weaver 1993). The direct impact of the fastest particles on the surrounding medium should produce a copious amount of $\gamma$-ray lines of $\mathrm{C}$ and $\mathrm{O}$, as observed in Orion (Fields et al. 1995). This case is particularly clean since there is no free parameter in the problem. The energy spectrum and the number of fast nuclei of the different kinds are derived directly from the calculated velocity and composition profiles of the external part of 
the supernova. The spectrum is close to a power law with index 4 and a maximum energy $\sim 10 \mathrm{MeV} / \mathrm{n}$. Here the irradiation time $\tau$ is not a free parameter, but is identified with the stopping time of the nuclei ejected imposed by ionization losses in the cloud medium (i.e. a few $10^{3} \mathrm{yrs}$ ). Since the exposure time is fixed, we may not adjust it, as done in the previous section, to ensure that the light element yields make a significant contribution to LiBeB evolution; instead the yields are fixed. Unfortunately, due to the shorter irradiation time compared to the WC case, the LiBeB yields obtained are of order $10^{-9} \mathrm{M}_{\odot}$ (compared to typical $\nu$-process yields of $\sim 10^{-7} \mathrm{M}_{\odot}$ ) and fall short of explaining the observations.

On the other hand, this kind of process could serve as excellent injector to subsequent acceleration. In such a scenario, shock acceleration of the material ejected by SN (as well as stellar winds) is required to get efficient production of light elements by $\mathrm{C}-\mathrm{O}$ rich ejecta (see the following section). If further acceleration of SN and wind material does not take place at all (which is unlikely, see e.g. Nath \& Biermann 1994; Biermann 1995; Bykov \& Bloemen 1994), then to fit B in the early galaxy demands the full contribution of neutrino production $(f=1)$. But in this case, ${ }^{9} \mathrm{Be}$ is underproduced in the early galactic times. The overconfinement scenario (Prantzos et al. 1993) being excluded since it leads to a slope of 2, a complementary process of primary nature is required (see e.g. Tayler, 1995), which would probably bring its own boron contribution. Consequently, once again, the neutrino contribution would have to be reduced.

\subsection{Production by reaccelerated supernova ejecta}

We consider now the contribution of all supernovae $\left(15-100 \mathrm{M}_{\odot}\right.$; Weaver \& Woosley 1993; Woosley, Langer \& Weaver 1993) assuming (re)acceleration in their ejecta with the same spectrum the case of the direct ejecta $(n=4)$, but pushing the maximum energy up to $30 \mathrm{MeV} / \mathrm{n}$ instead of $10 \mathrm{MeV} / \mathrm{n}$. This choice of spectrum parameters does not contradict any observational constraint. As with the WC case (\$3.1), the composition and irradiation (reacceleration) time remain free parameters.

Adopting these spectral characteristics, we have varied the lower mass limit of the contributing supernovae from $m_{\text {inf }}=15$ to $60 \mathrm{M}_{\odot}$. As expected, the lower $m_{\text {inf }}$, the higher is 
the average yields, and the shorter $\tau$. Further, $\tau$ varies between 500 yrs $\left(M>15 \mathrm{M}_{\odot}\right)$ and 50 000 yrs, $\left(M>60 \mathrm{M}_{\odot}\right)$. The supernovae of lower mass $\left(M<25 \mathrm{M}_{\odot}\right)$ are so efficient that they leave no room for the $\nu$-process or GCR contributions, which is quite unrealistic; thus we are again driven to favor the massive stars alone as sources $\left(\mathrm{M}\right.$ above $\left.35 \mathrm{M}_{\odot}\right)$. For the mass spectrum chosen in $\S 3.1\left(M>60 \mathrm{M}_{\odot}\right)$, the ${ }^{11} \mathrm{~B} /{ }^{10} \mathrm{~B}$ ratio is about 3 instead of 4 and a slight neutrino contribution is allowed as in the previous case $(f=0.2)$. In this specific situation, an important part of the solar system lithium, if not all, can be produced by LEN in the disk due to the operation of the $\alpha+\alpha$ reaction. Indeed, more ${ }^{6} \mathrm{Li}$ and ${ }^{7} \mathrm{Li}$ are produced than in the case of the most massive stars $\left(M>60 \mathrm{M}_{\odot}\right)$ since supernovae between 35 and $60 \mathrm{M}_{\odot}$ are more helium rich than above this mass (Weaver \& Woosley 1993).

\subsection{Implications for the $\nu$-Process}

If the LEN contribution is a significant source of Be in the early Galaxy, then it necessarily produces copious B as well. Thus to avoid B overproduction in early and solar epochs, the $\nu$-process yields are required to be reduced from their calculated values. Is such a reduction plausible? To be sure, supernovae are known to create a huge neutrino flux, as observed in SN 1987A. However, the details of the neutrino spectrum are more model-dependent. The largest uncertainties lie in the neutrino temperature (which controls the neutrino flux) and the neutrino spallation cross-section. Both sources carry at least a factor of two uncertainty (Woosley 1995, private communication). The LEN spectrum with $E_{c}=20 \mathrm{MeV}$, and a reduction in the $\nu$-process yields by a factor of five, is probably at the limit of compatibility between these two mechanisms. Note that if the $\nu$-process yields can be verified, (e.g., via

${ }^{19} \mathrm{~F}$ observations (Timmes, Woosley \& Weaver 1995), or through a confirmation of the details of the model parameters used to get the Li and B yields) then there is a potential conflict with the LEN hypothesis. In this case, a new source of primary ${ }^{9}$ Be would be required. 


\section{Conclusion}

We have estimated the contribution of the different spallation processes (nuclei at high and low energies and neutrinos at low energy) to the production and evolution of the LiBeB isotopes. Low energy nuclei are required to explain the Orion $\gamma$-ray observations, and must have composition that is overabundant in $\mathrm{C}$ and $\mathrm{O}$. Examining specific sources, we find that nuclei reflecting the composition of $\mathrm{C}$ and $\mathrm{O}$ rich stellar ejecta (WC and/or massive undressed $\mathrm{SN})$, moderately accelerated in the source vicinity by shock waves or turbulence, would play a significant role in the story of Be and B. Depending on the spectrum chosen, there is a varying amount of room for the GCR and neutrino processes; but regardless of the spectrum the low-energy nuclei contribution is important. This unique process is able to explain consistently the Be and $\mathrm{B}$ evolution and solar system abundances (using the meteoritic $\mathrm{B} / \mathrm{H}$ ratio), and the meteoritic ${ }^{11} \mathrm{~B} /{ }^{10} \mathrm{~B}$ ratio, with the GCR playing a secondary role, especially at low metallicity. A more detailed work, involving updated GCR production rates and a more refined analysis of the different components will be presented in a forthcoming paper (Lemoine et al. 1995).

While WC-type sources provide particle compositions that are both good candidates for Orion and which give good $\mathrm{LiBeB}$ evolution, direct ejecta seem to do only the former; their LiBeB production is negligible due to the short particle lifetime (ionization stopping time). With reaccelerated ejecta, the LEN component can again provide a key component in a good fit to LiBeB evolution, but only for source compositions like those of massive supernovae. Thus we see that, at least for the cases we have studied, massive progenitors for LEN particles are needed to provide a good LiBeB evolution. The details of such a selection effect remain to be shown; our results provide a hint to model-builders.

Since we have to reduce $\left(E_{c}=20 \mathrm{MeV} / \mathrm{n}\right)$ the neutrino yields by a factor 5 even in the best case, we fall short explaining Li in population I stars. In this case, stellar sources are required as AGB stars (Abia, Isern and Canal 1993). They should be rather long living (small masses) otherwise the Spite plateau would be violated. Similarly, even if $\nu$-process nucleosynthesis is the dominant mechanism in the production of ${ }^{11} \mathrm{~B}$, the ${ }^{11} \mathrm{~B} /{ }^{10} \mathrm{~B}$ ratio necessitates a reduction in the neutrino spallation yields and would also preclude the $\nu$-process from explaining the 
population I Li abundances (Olive et al. 1994).

In closing, it is worth noting that the solar $\mathrm{B} / \mathrm{H}$ ratio is still uncertain, varying from $2 \times 10^{-10}$ (within a factor of 2 for Pop I stars, Boesgaard and Heacox 1978) and (7.5 \pm 0.6$) \times$ $10^{-10}$ in carbonaceous chondrites (Anders and Grevesse 1989), to the photospheric value of $4 \times 10^{-10}$ (Khol et al. 1977). As a byproduct of this work, we remark that in all the fits the meteoritic $\mathrm{B} / \mathrm{H}$ value is favored. The progress in this field is now linked to better abundance determination.

We are grateful to Tom Weaver, Stan Woosley, and Frank Timmes for access to the neutrino yields and the latter two for helpful conversations. We thank Yvette Oberto and Roland Lehoucq for permanent help. This material was based on work supported by the North Atlantic Treaty Organization under a grant awarded in 1994. This work was supported in part by PICS n¹14, "Origin and evolution of the light elements," CNRS. This work was also supported in part by DOE grant DE-FG02-94ER-40823.

\section{References}

Abia, C., Isern, J. \& Canal, R. 1993, A\&A. 275, 96

Anders, E. \& Grevesse, N. 1989, Geochim. et Cosmochim. Acta, 35, 197

Biermann, P.L. 1995, in Cosmic Winds and Heliospheres, ed. J.R. Jokipii, C.P. Sonnet \& M.S. Giampapa

Bloemen H. et al. . 1994, A\&A, 281, L5

Boesgaard, A.M. \& Heacox, H.D. 1978, Ap.J., 226, 888

Boesgaard A. \& King J.R. 1993, AJ, 106, 2309

Bykov, A. \& Bloemen, H. 1994, A\&A, 283, L1

Cassé, M. Lehoucq, R. \& Vangioni-Flam, E. 1995, Nature, 373, 318

Chaussidon, M. \& Robert, F. 1995, Nature, 374, 337 
Duncan D., Lambert D. \& Lemke M. 1992, ApJ, 401, 584

Duncan, D. et al. . 1995, Maryland conference : Cosmic Abundances, in press

Fields, B.D., Cassé M. \& Vangioni-Flam, E., Nomoto K., 1995a, ApJ, in press

Fields, B.D., Olive, K.A., \& Schramm, D.N. 1995b, ApJ, 439, 854.

Gilmore G., Gustafsson B., Edvardsson B. \& Nissen P.E. 1992, Nature 357, 379

Janka, H. Th.,Muller, E., 1995, A\&A, submitted

Kiselman, D. 1994, A\&A, 286, 169

Kiselman, D. \& Carlsson, M. 1994, in The Light Element Abundances, ed. P. Crane, 372

Kohl, J.L., Parkinson, W.H. \& Withbroe, N. 1977, ApJ, 212, L101

Lemoine, M., Vangioni-Flam, E. \& Cassé, M., 1995, in preparation

Maeder A. 1992, A\&A, 264, 105

Nath, B.B. \& Biermann, P.L. 1994, MNRAS, 270, L33

Nomoto, K., Iwamoto,K. \& Suzuki, T. 1995, Physics Reports, in press

Olive K.A., Prantzos N., Scully S. \& Vangioni-Flam E. 1994, ApJ, 424, 666

Prantzos, N., Cassé,M. \& Vangioni-Flam,E. 1993, Ap.J., 403, 630

Ramaty R., Kozlovsky B. \& Lingenfelter R.E., 1995a, ApJ, 438, L21

Ramaty R., Kozlovsky B. \& Lingenfelter R.E. 1995b, in Proceedings of the 17th Texas Symposium, New York Acad. Sci., in press

Ramaty, R., Kozlovsky, B. \& Lingenfelter, R.E. 1996, ApJ, in press

Read, S.M.,\& Viola,V.E., 1984, Atomic Data and Nuclear Data Tables, 31, 359

Rebolo, R., Molaro, P., Beckman J.E. 1988, A\&A, 192, 192

Rebull, L.M. et al. , 1995, Maryland conference: Cosmic Abundances, in press

Ryan, S., Bessell, M., Sutherland, R.\& Norris, J. 1990, ApJ, 348, L57

Ryan S., Norris I., Bessel M. \& Deliyannis C. 1994, ApJ, 388, 184

Shima, S.and Honda, W., 1962, J. Geophys. Res., 68, 2849 
Spite F. \& Spite M. 1993, "Origin and Evolution of the Elements, ed. N. Prantzos, E. Vangioni-Flam \& M. Cassé, Cambridge University Press, 201

Tatischeff, V. et al. 1996, in preparation

Tayler, R.J. 1995, MNRAS, 273, 215

Thevenin, F. et al. , 1996, in preparation

Timmes, F.X., Woosley, S.E. \& Weaver, T.A. 1995, ApJ in press

Vangioni-Flam E., Cassé M. \& Ramaty, R. 1995, ApJ, submitted

Weaver T. M. \& Woosley S.E. 1993, Phys. Rep. 227, 65

Woosley,S.E., Hartmann,D., Hoffman, R., Haxton, W. 1990, ApJ., 356, 272

Woosley, S. E., Langer, N. A., Weaver T. A. 1993, ApJ, 411, 823

Woosley, S.E. \& Weaver, T.A. 1995, ApJ, submitted 
Table 1: LiBeB Production Sites by Isotope

\begin{tabular}{cccccc}
\hline \hline & \multicolumn{5}{c}{ Isotope } \\
Source & ${ }^{6} \mathrm{Li}$ & ${ }^{7} \mathrm{Li}$ & ${ }^{9} \mathrm{Be}$ & ${ }^{10} \mathrm{~B}$ & ${ }^{11} \mathrm{~B}$ \\
\hline LEN & yes & yes & yes & yes & yes \\
GCR & yes & yes & yes & yes & yes \\
$\nu$-process & no & yes & no & no & yes \\
\hline \hline
\end{tabular}

Table 2: The Interplay of Source Parameters and Observables

\begin{tabular}{ccc}
\hline \hline Source & Parameter & Fixed by \\
\hline LEN: steady state & irradiation time $\tau$ & $\mathrm{Be} / \mathrm{H}$ at $[\mathrm{Fe} / \mathrm{H}] \leq-1$ \\
LEN: direct ejecta & none & $\mathrm{N} / \mathrm{A}$ \\
LEN: reaccelerated ejecta & lower mass limit $m_{\text {inf }}$ & varies \\
GCR & total flux $F$ & solar $\mathrm{Be} / \mathrm{H}$ \\
$\nu$-process & yield normalization $f$ & solar ${ }^{11} \mathrm{~B} /{ }^{10} \mathrm{~B}$ \\
\hline \hline
\end{tabular}




\section{Figure Captions}

Figure 1: Evolution of ${ }^{7} \mathrm{Li} / \mathrm{H}$ from neutrino spallation only.

The metallicity dependent yields from Woosley and Weaver (1995) are multiplied by factors $f=1$ (dotted line), $f=0.5$ (full line). Data points are from Spite and Spite (1993), Rebolo et al. (1988).

Figure 2: Evolution of $\mathrm{B} / \mathrm{H}$ from neutrino spallation only.

Same as fig. 1. Data points from Duncan et al. (1992) and Kiselman \& Carlsson (1994). The three values at solar $[\mathrm{Fe} / \mathrm{H}]$, from bottom to top correspond to solar photospheric (Khol et al. 1977), present galactic average (Boesgaard and Heacox 1978) and meteoritic (Anders and Grevesse 1989).

Figure 3: Evolution of ${ }^{11} \mathrm{~B} /{ }^{10} \mathrm{~B}$.

Full line: LEN $\left(E_{c}=30 \mathrm{MeV} / \mathrm{n}\right)+$ GCR; dotted line: LEN $\left(E_{c}=20 \mathrm{MeV} / \mathrm{n}\right)$ $+\mathrm{GCR}+$ neutrinos $(f=0.2)$.

Figure 4: Evolution of B/Be.

same as figure 3 .

Figure 5 : Evolution of Be and B.

LEN $\left(E_{c}=20 \mathrm{MeV} / \mathrm{n}\right)+\mathrm{GCR}+$ neutrinos $(f=0.2)$. Data points are from Ryan et al. (1990), Gilmore et al. (1992), Ryan et al. (1994) and Boesgaard and King (1993) (Be), Duncan et al. (1992) and Kiselman \& Carlsson (1994) (B). 


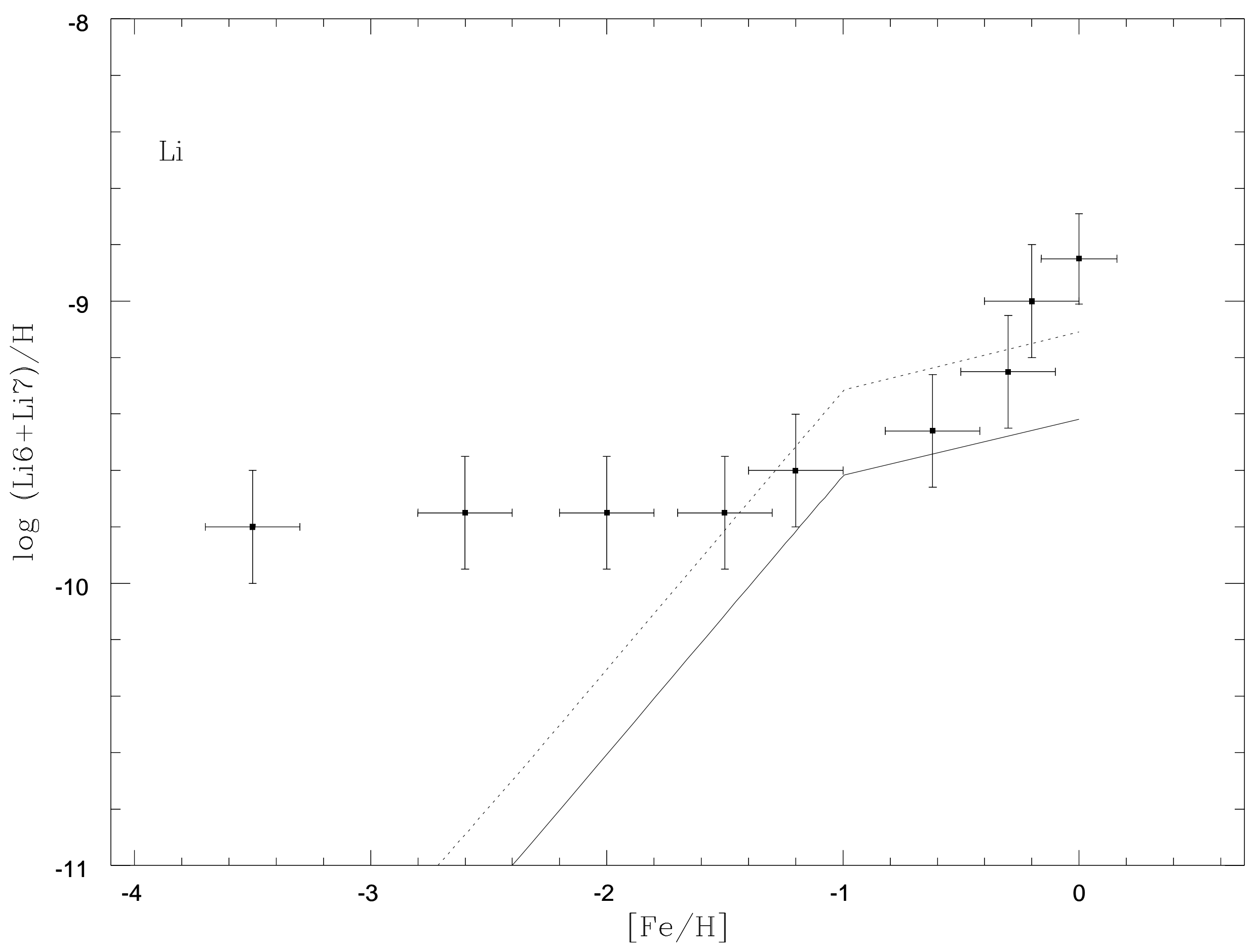




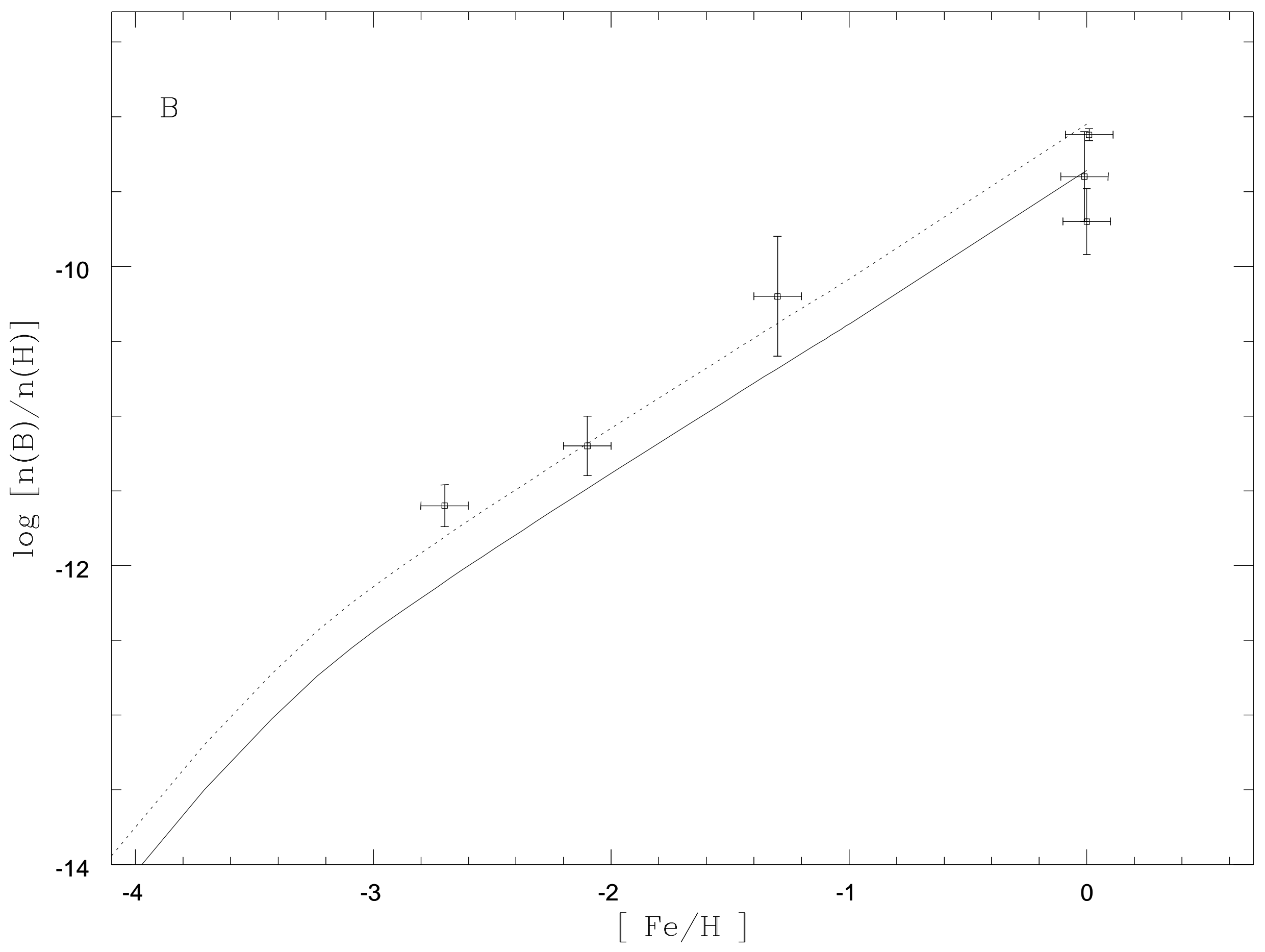




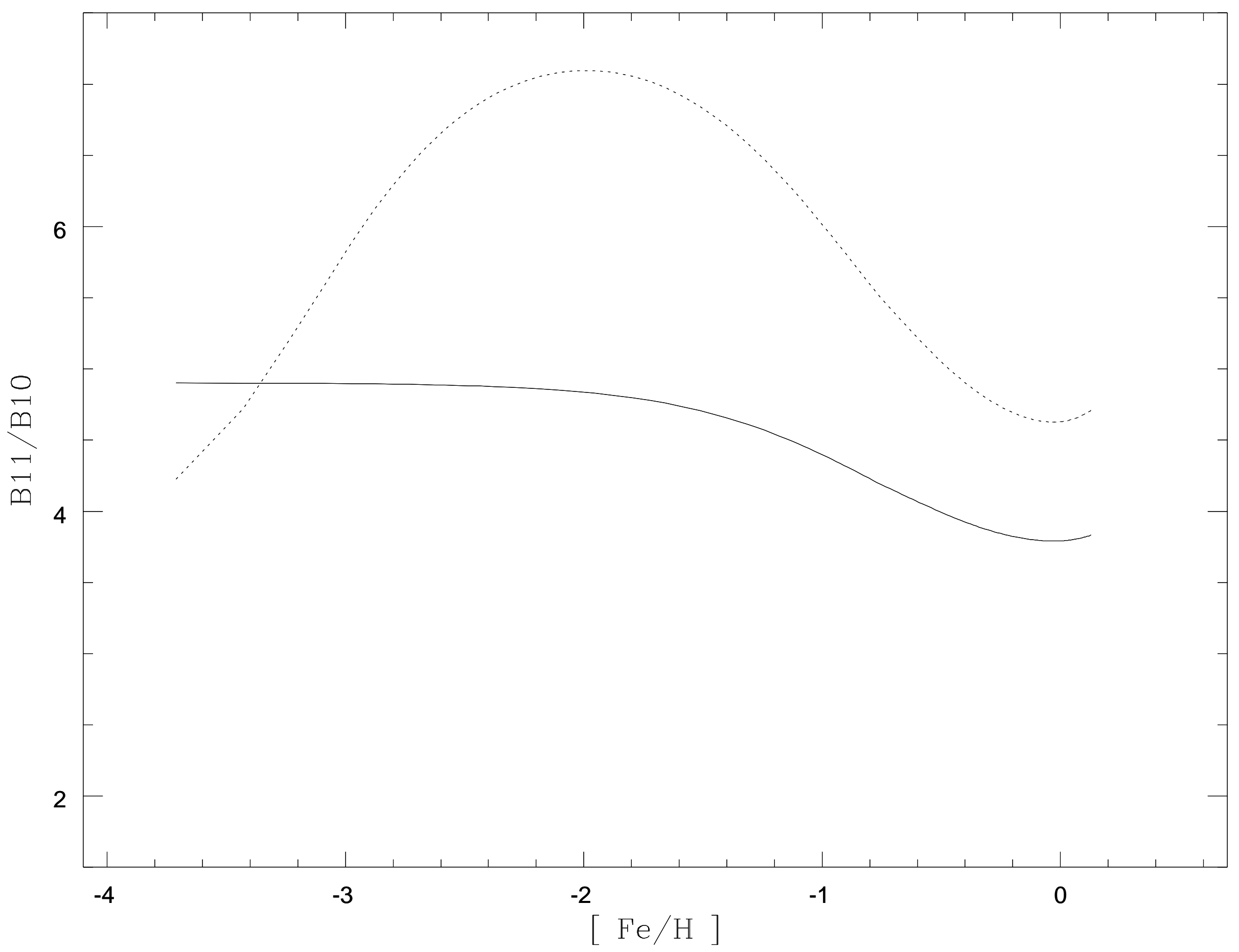




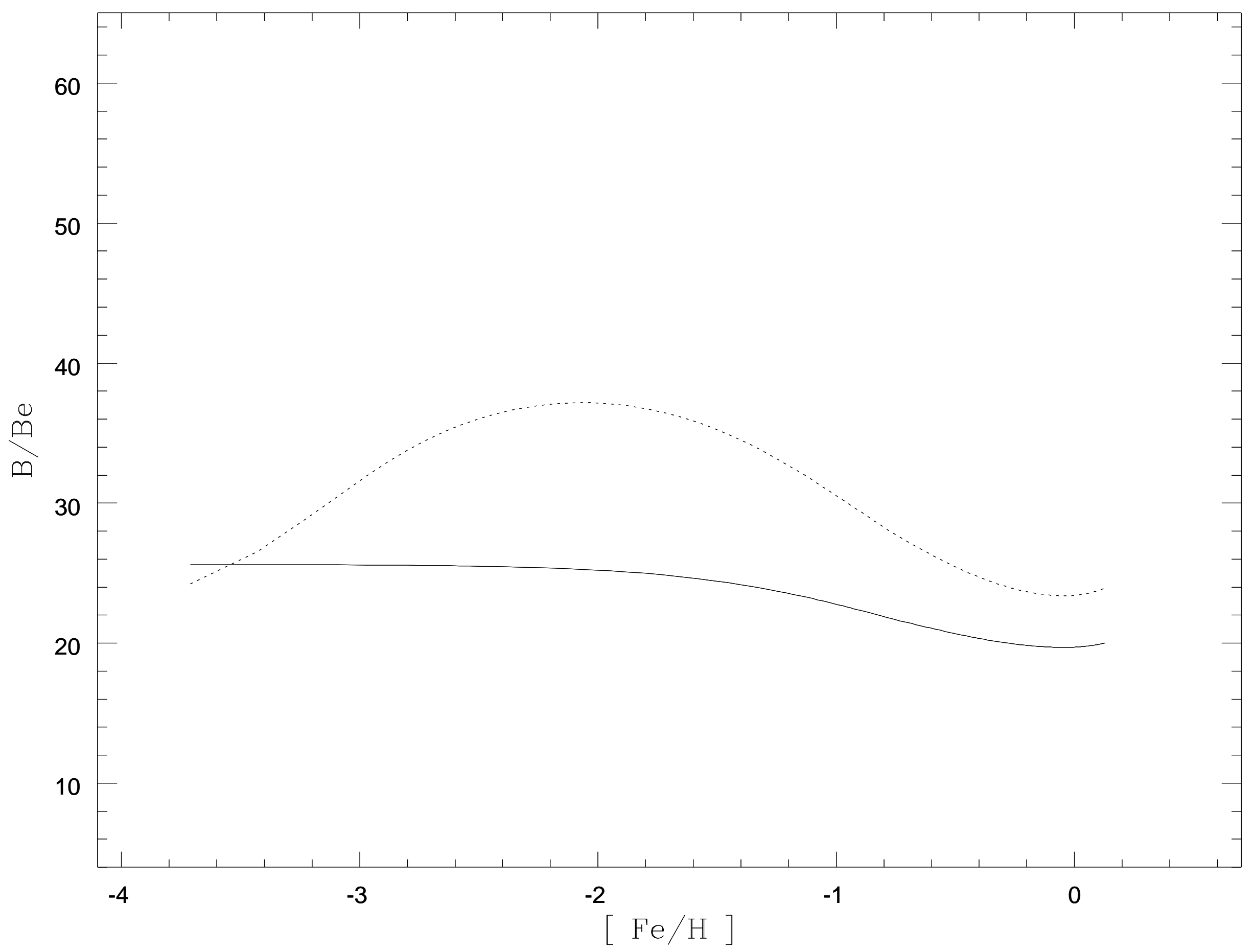




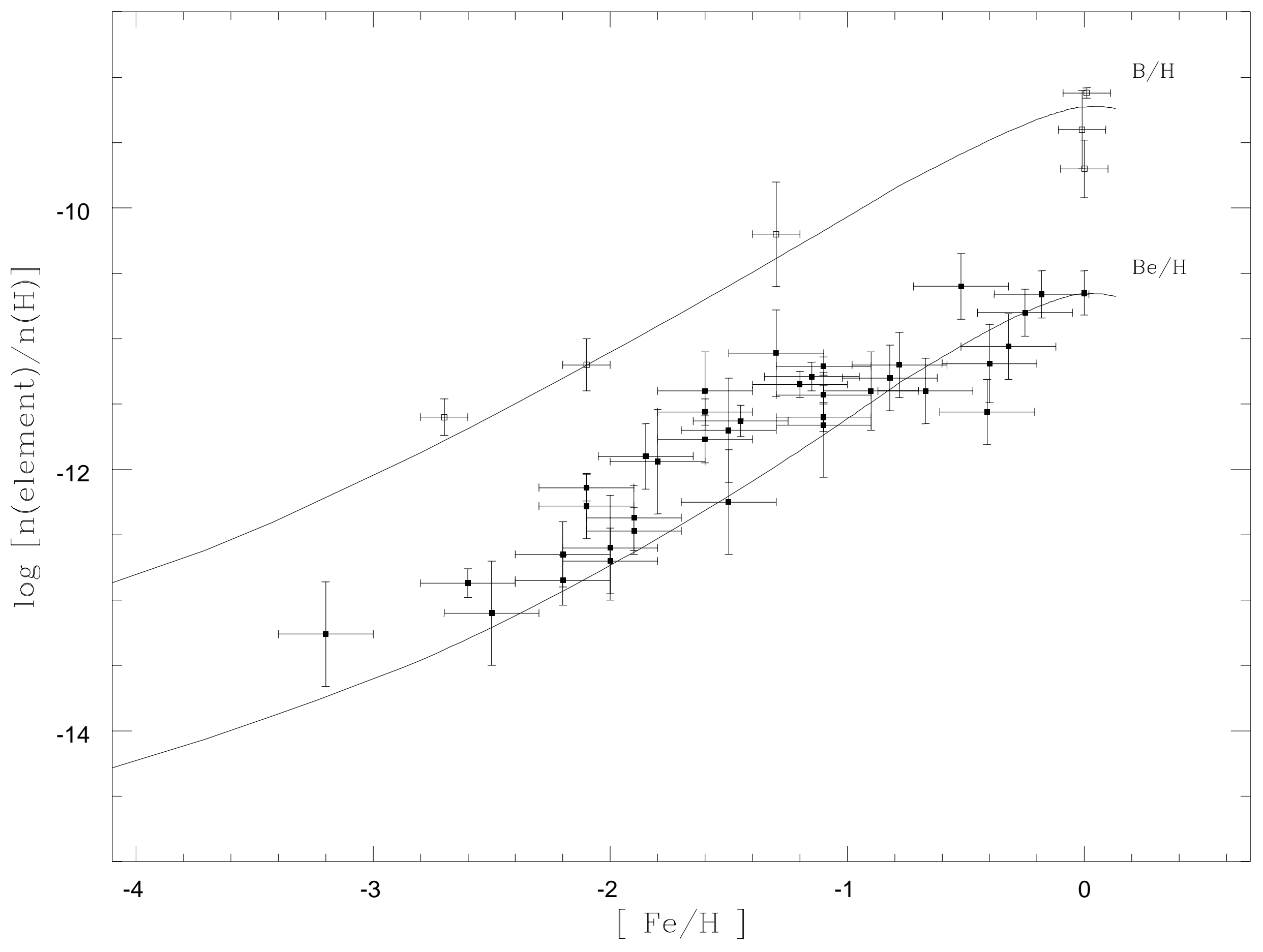

